\title{
Aberrant Origin of Radial Artery in Arm
}

\author{
Vivek Sathe ${ }^{1}$, Rishi Pokhrel
}

${ }^{1}$ Department of Anatomy, Nepalese Army Institute Of Health Sciences, Kathmandu, Nepal.

\begin{abstract}
During dissection in middle-aged male cadaver, an aberrant radial artery was noticed in the left arm. The artery was taking origin from the upper part of the brachial artery i.e. at a point when axillary artery leaves the axilla and becomes brachial artery.

Course of the aberrant vessel was studied in the arm and the forearm. In the upper part of arm the vessel took origin on the lateral side of brachial plexus trunks and traveled to the lateral region of the cubital fossa travelling lateral to the biceps brachii and deep to skin, superficial and deep fascia. In the lower part of the arm i.e. just above the cubital fossa, aberrant vessel was lateral and entered the forearm deep to the pronator teres. Throughout its course the vessel laid superficial to forearm muscles, it was covered by the skin superficial and deep fascia. At the wrist its position was normal i.e. against anterior border of the radius.
\end{abstract}

Keywords: aberrant; origin; radial artery.

\section{INTRODUCTION}

Brachial artery divides into its terminal ulnar and radial branches at the level of neck of radius or one $\mathrm{cm}$ distal to the flexor crease of elbow. Radial artery is smaller branch and is the direct continuation of the brachial artery ${ }^{1}$.

Radial artery at its origin is overlapped by the anterior belly of the Brachioradialis muscle. In the remaining forearm the vessel lie below the skin, superficial fascia and deep fascia. Just above the wrist it lies against the anterior border of radius where its pulsations are felt ${ }^{1}$.

\section{CASE REPORT}

During routine human cadaveric dissections carried out at our department; an aberrant vessel was noticed in the left arm of a middle-aged male cadaver.

The aberrant artery (figure 1) was taking origin from the brachial artery at a point where axillary artery leaves axilla and becomes brachial artery. From the course of the vessel in the arm and fore arm the vessel was identified as an aberrant radial artery. Instead of its normal site of origin i.e. at the neck of the radius, the artery has taken origin from the brachial artery in the upper third of the arm. The fact was confirmed in cubital fossa where ulnar artery was continuation of brachial and no radial division was found

During the study of course of the aberrant radial artery, it was observed that it gave no branches in the arm and the forearm. The vessel had smaller caliber at origin than ulnar artery.

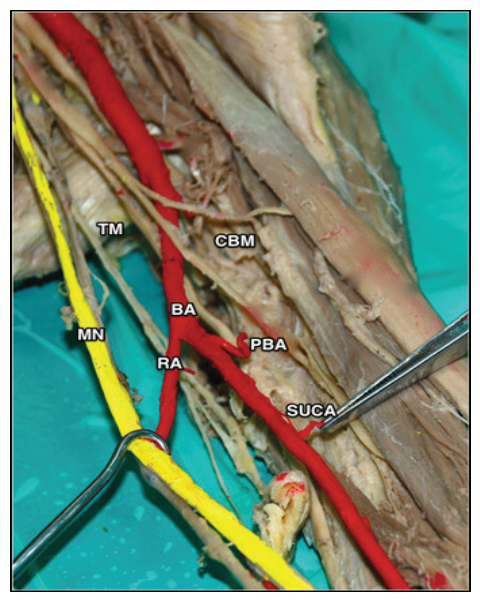

Figure 1: High origin of brachial artery; TM: teres major, CBM: coracobrachialis muscle, MN: Median nerve, BA: brachial artery, RA: radial artery, PBA: profonda brachii artery, SUCA: superior ulnar collateral artery

\section{Correspondence:}

Rishi Pokhrel

Department of Anatomy, Nepalese Army Institute of Health Sciences.

Email: rongon28us@yahoo.com 


\section{DISCUSSION}

\section{Origin of forearm vessels}

Embryologically, limbs develop from the trunk region of the embryo. Upper limb bud originate from the lateral aspect of the trunk on the day 26 to 28 and completely differentiates in to shoulder, arm, forearm and hand by the 25 day of embryonic life ${ }^{1}$.

Limb bud is a mesenchymal out growth from the parietal or somatic mesoderm covered with tall columnar epithelium developing from the embryonic ectoderm. From parietal mesoderm develops connective tissues of the limbs connective tissue (dense and loose), cartilages, bones and tendons. Muscles and blood vessels of the limbs develop from the myotomic somites of the paraxial mesoderm ${ }^{2}$.

In the mesenchyme of the limb bud capillary plexus develops in the myotomic somites. Arterial capillary bed receives blood supply from intersegmental arteries from somite C5 to T-I or T-2. Cells in the mesenchyme have inducting effect, which develops info tunica intima of the capillaries ${ }^{3}$ Figure 2: Origin of axis artery of upper limb from 7 th intersegmental artery.

Subclavian artery is the axial artery of the upper limb (figure 2). In the right limb subclavian originates from the arch of aorta as seventh segmental artery. The vessel enters the limb as axillary artery continues in arm as brachial, in the forearm anterior interosseous artery and in the palm as deep palmar arch. Radial and ulnar arteries are the latest branches to appear in the forearm. Initially radial artery takes origin in the arm and supply flexor group of arm muscle mainly the biceps brachii. Later on radial artery makes new

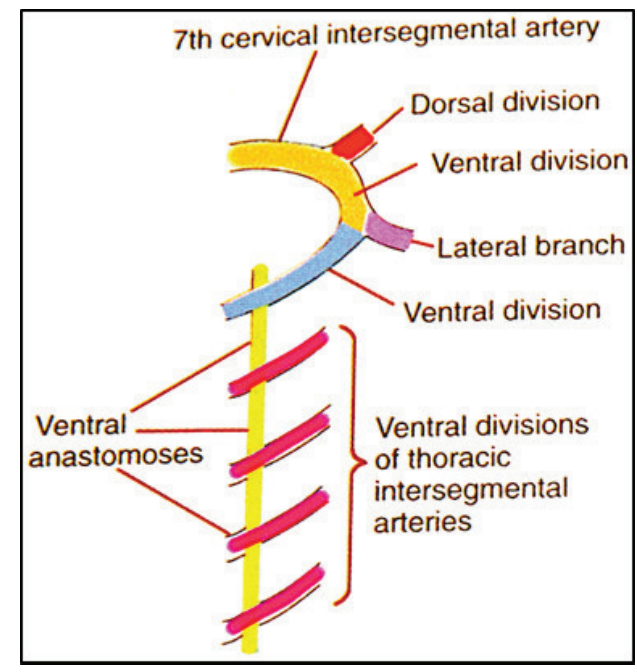

connection with the brachial artery at the level of neck of the radius. Upper portion of the radial artery disappears.

\section{Point of dispute}

Many authors regard radial artery as the continuation of the brachial but for the mode of development radial joins the brachial at a later date. Ulna branch should be regarded as the main continuation of the brachial.

\section{CONCLUSION}

In the present case report initial connection of radial artery with the brachial still persisted in the adult. It has failed to anastomose with the brachial at the neck of the radius.

As a result of this, it has changed its course in the forearm i.e. uncovered by the anterior head of Brachioradialis. For this reason the aberrant artery is the radial artery having an aberrant origin.

\section{REFERENCES}

1. Standring S, Borley NR. Gray's Anatomy: The Anatomical Basis of Clinical Practice: Churchill Livingstone/Elsevier; 2008.

2. Mathes SJ, Nahai F. Classification of the vascular anatomy of muscles: experimental and clinical correlation. Plastic and reconstructive surgery. 1981;67(2):177-87.

3. Langille RM, Hall BK. Developmental processes, developmental sequences and early vertebrate phylogeny. Biological Reviews. 1989;64(2):7391. 